\title{
Experimental setup for high resolution X-ray spectroscopy of solids and liquid samples
}

\author{
Zhong Yin ${ }^{\mathrm{a}, \mathrm{b}}$, Ivan Rajković ${ }^{\mathrm{a},{ }^{*}}$, Dirk Raiser ${ }^{\mathrm{a}}$, Mirko Scholz ${ }^{\mathrm{a}}$ and Simone Techert ${ }^{\mathrm{a}, \mathrm{b}, \mathrm{c}}$ \\ ${ }^{a}$ Max Planck Institute for Biophysical Chemistry, Structural Dynamics of (Bio)chemical Systems, \\ 37077 Göttingen, Germany \\ ${ }^{b}$ Deutsches Elektronen-Synchrotron DESY, Structural Dynamics of (Bio)chemical Systems, \\ 22607 Hamburg, Germany \\ ${ }^{c}$ Institute for X-ray Physics, Georg-August-University Göttingen, 37077 Göttingen, Germany
}

\begin{abstract}
Here we present a next-generation experimental setup for high-resolution X-ray spectroscopy of solid and liquid samples in the soft X-ray region to elucidate the complex molecular structures of (bio)chemical systems. The setup consists of a main target chamber, a target holder for either solid samples or a liquid jet delivery system, and a high-resolution soft Xray grating spectrometer. This setup is in commissioning at PETRA III, presently one of the most brilliant storage ring based X-ray radiation sources in the world. The newly designed grazing incidence grating spectrometer is utilized for high-resolution measurement in the XUV range from $1 \mathrm{~nm}$ up to $6 \mathrm{~nm}$.
\end{abstract}

\section{INTRODUCTION}

X-ray emission spectroscopy (XES) is an ideal tool to study the local electronic structure and its surroundings of condensed matter as well as chemical and biological systems. In detail, this method probes the occupied electrons in an atomic or molecular system. This can be done in two ways, resonantly or non-resonantly. In the non-resonant case, the excitation is tuned to ionize the core-hole electron and the following decay is measured. In the resonant process (resonant inelastic X-ray scattering, RIXS) the core-hole electron is excited to an unoccupied orbital, making the excitation and the following fluorescence coupled events. Thus the X-ray fluorescence contains ultrafast time information over the range of the core-hole lifetime. Overall, XES is element, orbital, polarization and bulk sensitive [1,2].

But there have been scientific and technical challenges associated with this method. The radiation source needs to be in the X-ray energy range and must be tunable with high X-ray energy resolution. The K-edge electrons of the life important elements like C, N, O are energetically located in the water window of $280-530 \mathrm{eV}$. Therefore, the radiative decay is up to several magnitudes lower compared to the non-radiative decay channel, making a highly brilliant X-ray source essential. The fast development of brilliant synchrotron facilities and Free Electron Lasers (FEL) as a monochromatic source for soft X-ray made X-ray emission spectroscopy a feasible method to study the electronic structure of elements in the water window. Another challenge is the study the chemical and biological systems in their natural environment, i.e. in the liquid phase. To overcome the instability of liquids in vacuum, two concepts proved to be reliable: the liquid cell and the liquid jet [3,4]. In a cell a $100 \mathrm{~nm}$ thin silicon nitride $\left(\mathrm{Si}_{3} \mathrm{~N}_{4}\right)$ or carbon membrane

"present address: Paul Scherrer Institute, CH-5232 Villigen PSI, Switzerland

X-Ray Lasers and Coherent X-Ray Sources: Development and Applications X, edited by Annie Klisnick, Carmen S. Menoni, Proc. of SPIE Vol. 8849, 88490I · (c) 2013 SPIE

CCC code: $0277-786 X / 13 / \$ 18 \cdot$ doi: $10.1117 / 12.2023992$

Proc. of SPIE Vol. $8849884901-1$ 
separates the sample from the vacuum. However, using a membrane prevents the investigation of samples containing the same elements due to overlapping X-ray fluorescence of sample and membrane. Also the interactions of the membrane with the X-ray source and the fluorescence can affect the spectra and lead to a loss of photons and a longer data acquisition time. An alternative method to study systems in the liquid phase is the liquid jet developed by M. Faubel et al [3]. First this kind of liquid jet has been adapted for photoelectron spectroscopy [5].

The liquid jet offers the advantage of pure sample surface in vacuum environment with no interaction of the sample, $\mathrm{X}$-ray or fluorescence with the membrane, resulting in a higher photon flux and shorter data acquisition time.

In this paper, we report a new experimental endstation at the soft X-ray beamline of the most brilliant synchrotron source to date, PETRA III in Hamburg, Germany. The current design is also based on experiences from previous similar setups [6-8]. The new endstation consists of a sample holder for both liquid and solid samples and an X-ray detector. To detect the X-ray fluorescence a new grazing incidence grating spectrometer for X-ray wavelength from $1 \mathrm{~nm}$ up to $6 \mathrm{~nm}$ has been designed with a CCD detector. Additionally, a chemical reactor has been designed for inducing photochemical reactions in bulk sample systems.

\section{EXPERIMENTAL SETUP}

The scheme of the experimental setup is shown in fig 1 . The main components are the target chamber, where the X-ray fluorescence of the sample is produced, a target holder for both the liquid jet delivery system and solid samples, a photodiode in $45^{\circ}$ geometry with respect to the X-ray beam and a high-resolution grating spectrometer. Differential pumping stages separate the vacuum condition of the beamline (around $10^{-8} \mathrm{mbar}$ ) and the grating spectrometer (around $10^{-6} \mathrm{mbar}$ ) from the target chamber (around $10^{-3} \mathrm{mbar}$ ), thus ensuring no loss of photons which is an advantage over the similar setups using a foil. The liquid jet has a variable diameter around $15-30 \mu \mathrm{m}$. A GaAsP diode is placed at $45^{\circ}$ to the incoming X-ray beam and the liquid jet and is used to find the spatial overlap of the X-ray beam and the liquid jet. It also functions as a rapid method to record X-ray absorption spectra in total fluorescence yield (TFY) and to determine the excitation energies for XES or RIXS measurements. The target holder offers the space for solid samples as well as for liquid jet. The grating detector is installed in plane perpendicular to the incident beam. In the following sections the main parts of the endstation are described in more detail.

Proc. of SPIE Vol. $8849884901-2$ 


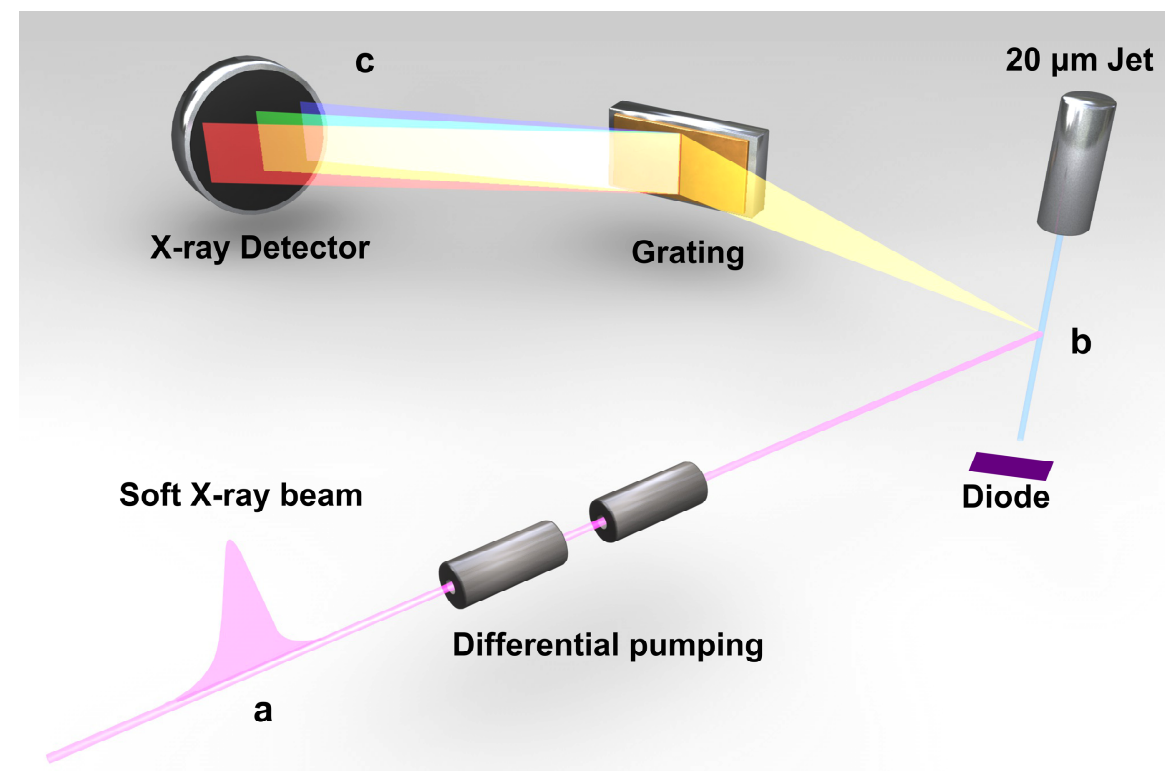

Fig. 1: Schematic drawing of the experimental setup. At a) the differential pumping stage is securing the vacuum conditions. The sample is introduced into the target chamber at b) and the X-ray fluorescence is recorded with a high-resolution grating spectrometer at c).

\subsection{Liquid Jet}

The sample delivery system consists of an HPCL pump, a degasser, a low pressure gradient unit (all from JASCO) and nozzles. The degasser removes the gaseous components of the sample, which could lead to instability of the jet. The low pressure gradient unit enables the mixing of up to four samples in any ratio from $1 \%$ up to $100 \%$ without stopping the running jet. The crucial part of the system is the HPLC pump, which delivers the sample to the target chamber.

The cylindrical nozzle diameter size used so far can vary from $15 \mu \mathrm{m}$ up to $30 \mu \mathrm{m}$, depending on the sample and experimental conditions. Theoretically, the nozzle size can cover a range from 5-100 $\mu \mathrm{m}$. Right below the exit of the nozzle, the laminar free flow can reach up to several $\mathrm{mm}$, depending on flow rate, before it breaks up into droplets. Besides having a real liquid surface in vacuum, the delivery sample system provides always fresh samples. No radiation damage is causing artifacts in the spectra, i.e., for a $50 \mu \mathrm{m}$ beam spot size and using a $20 \mu \mathrm{m}$ nozzle with a flow rate of $1 \mathrm{ml} / \mathrm{min}$, a repetition rate of up to $1 \mathrm{MHz}$ can be achieved without sample degrading. The potential repetition rate can go up to $2 \mathrm{MHz}$.

\subsection{Chemical Reactor}

A flexible chemical reactor (CR) was designed to study photochemical reactions, such as isomerization.

The CR can either be equipped with fluorescent tubes or with LEDs within a range from UV to infrared and replaces complex laser setups. The geometrical design of the CR assures a high light exposure. Additionally, cooling can be applied during the radiation process to avoid a high inversion rate for thermodynamically unstable samples. 


\subsection{High resolution grating spectrometer}

The newly developed grazing incidence grating spectrometer for the energy range of $270 \mathrm{eV}-1200 \mathrm{eV}$ is equipped with a concave variable line spacing flat field grating purchased from HITACHI (001-00659, BK7 substrate), a $\mu \mathrm{m}$ step-size resolution entrance slit and a position-sensitive detector. The entrance slit is adjustable in $\mu \mathrm{m}$ steps from $30 \mu \mathrm{m}$ down to $5 \mu \mathrm{m}$, depending on the favored resolution.

The average ruling density of the grating is 2400 grooves $/ \mathrm{mm}$ with a blaze angle of $3.15^{\circ}$ and a curvature radius of $\mathrm{R}=58542 \mathrm{~mm}$. The surface of the grating is gold coated, to ensure a high reflectivity of incoming X-rays. Fig. 2 shows the ray-traced spectral images and the focal curve characteristics for that grating. The variable line spacing (VLS) and concave design of the grating focuses the fluorescence on a line, which - compared to a cylindrical curvature with focus on the Rowland circle geometry - facilitates high resolution and prevents high order and geometrical aberrations on the spectra. A back-illuminated CCD X-ray camera from ANDOR is utilized for the detection of the X-ray fluorescence [9]. It consists of $2048 \times 512$ pixels with a pixel size of $13.5 \mu \mathrm{m} \times 13.5 \mu \mathrm{m}$.

\section{SPECTROMETER CHARACTERISTICS}

The high-resolution grating spectrometer is equipped with a blazed grazing incidence varied line spacing grating. Fig. 2 shows the theoretical focal point depending on the incident angle of the X-ray for the wavelength 1-6 nm. The $\mathrm{x}$ - axis is the distance between the grating and the detector, which keeps constant the distance between X-ray source or slit and the grating. The distance recommended by the manufacturer is $564 \mathrm{~mm}$. It should be noted that, due to the scattering characteristics, low wavelengths $\lambda_{1}$ are lower on the detector than the higher wavelength $\lambda_{2}$. At an incident angle of $89^{\circ}$ in respect to the surface normal of the grating, it can be seen that the focal point of a broad range of energy is covered. Keeping the $89^{\circ}$ incident angle and changing the source/slit to the grating distance leads to results, which are shown in Fig. 3. This figure shows the simulated focal point depending on the distance between source/slit and grating. The recommended distance of $564 \mathrm{~mm}$ is plotted with a deviation of $10 \mathrm{~mm}$. Fig. 4 shows the potential resolution power of the spectrometer in depending on the slit/source size. It can be noted that for lower energies a better energy resolution can be achieved than for higher energies. In experiment we expect to obtain a lower resolution, as the simulation is not considering e.g. the pixel size of the detector. The spectrometer allows adjusting the grating detector distance, so that a high spatial resolution is achieved on a wide range of energies. Furthermore, the incident angle is fixed at $1.0^{\circ}$ which is the optimal angle according to Fig 1. 


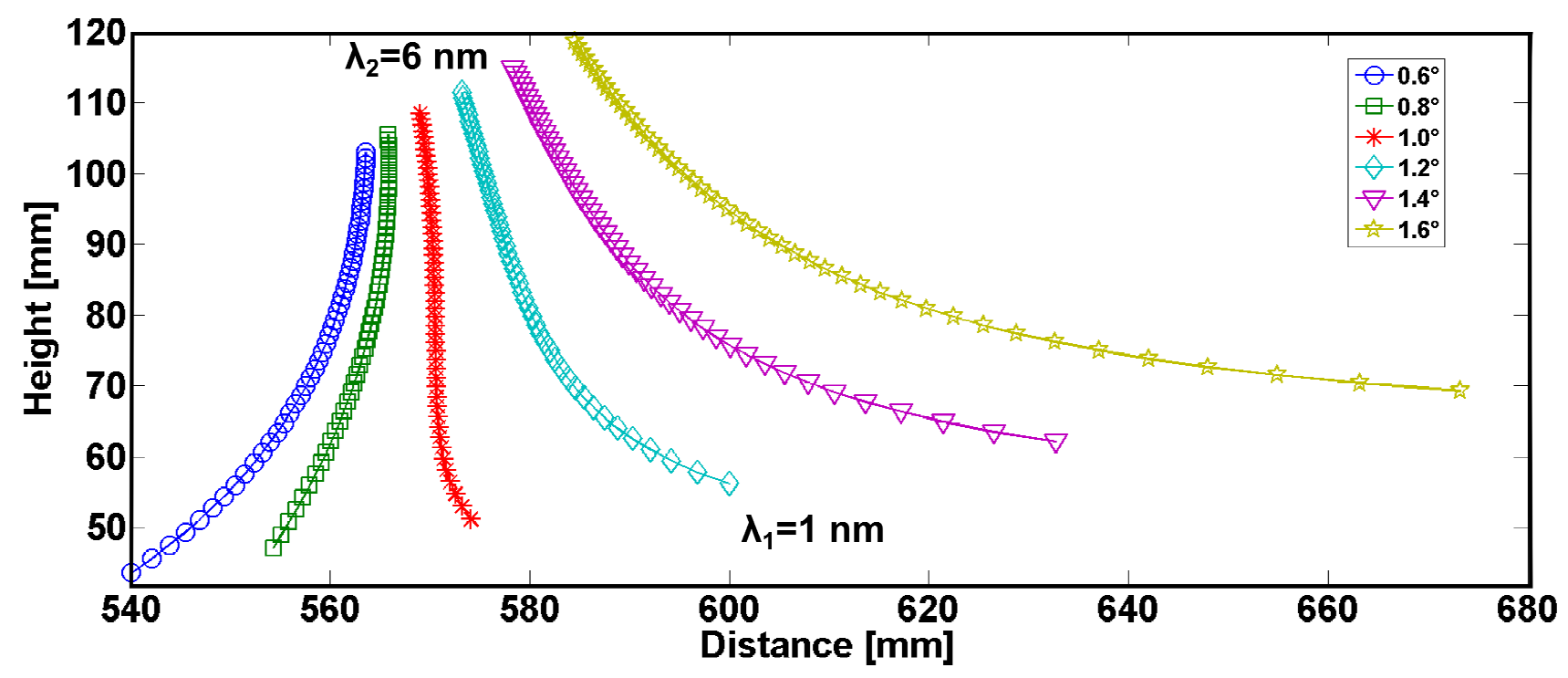

Fig. 2: The focal point depends on various incident angles. At the recommended value of $1^{\circ}$, the optimal grating to detector distance is minimal for the whole energy range of 1 to $6 \mathrm{~nm}$.

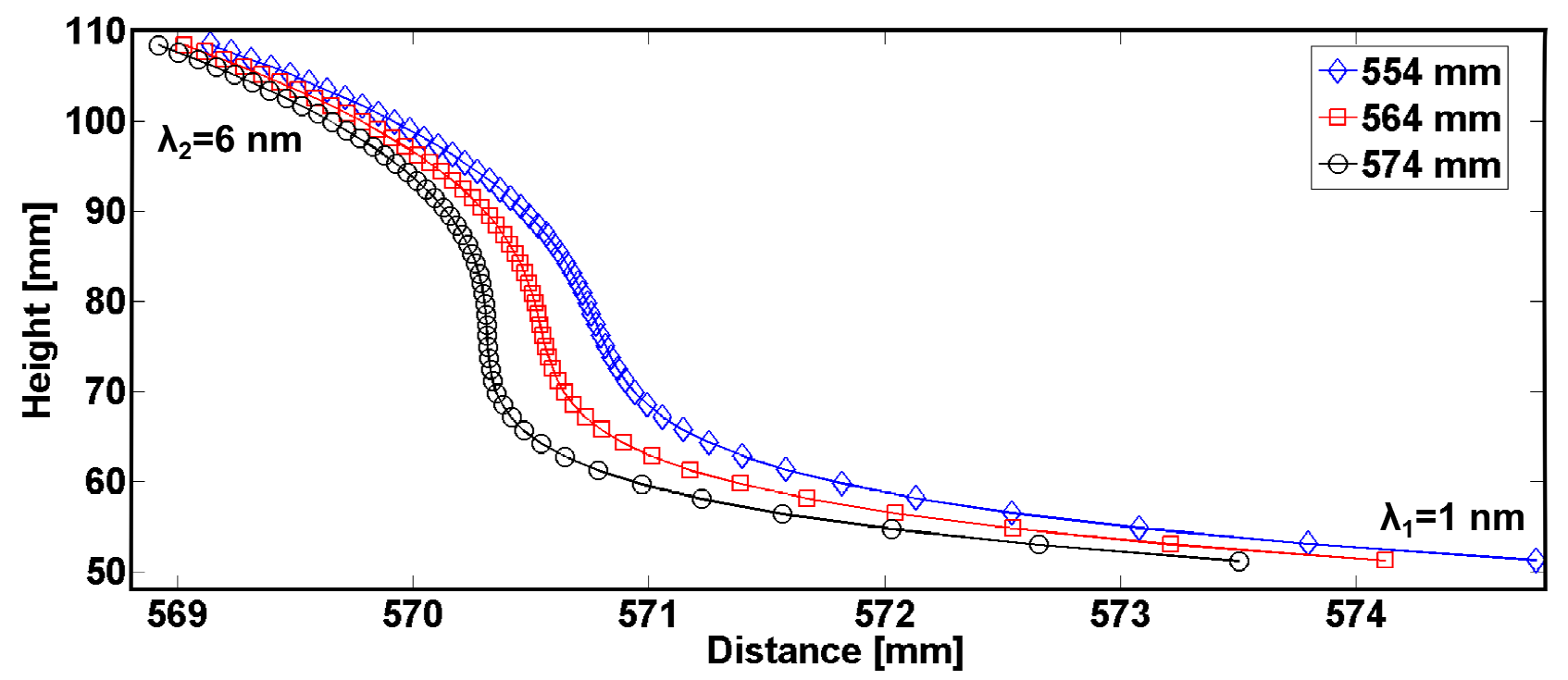

Fig 3: The focal behavior for the recommended distance from source/slit to grating and deviations from it. 


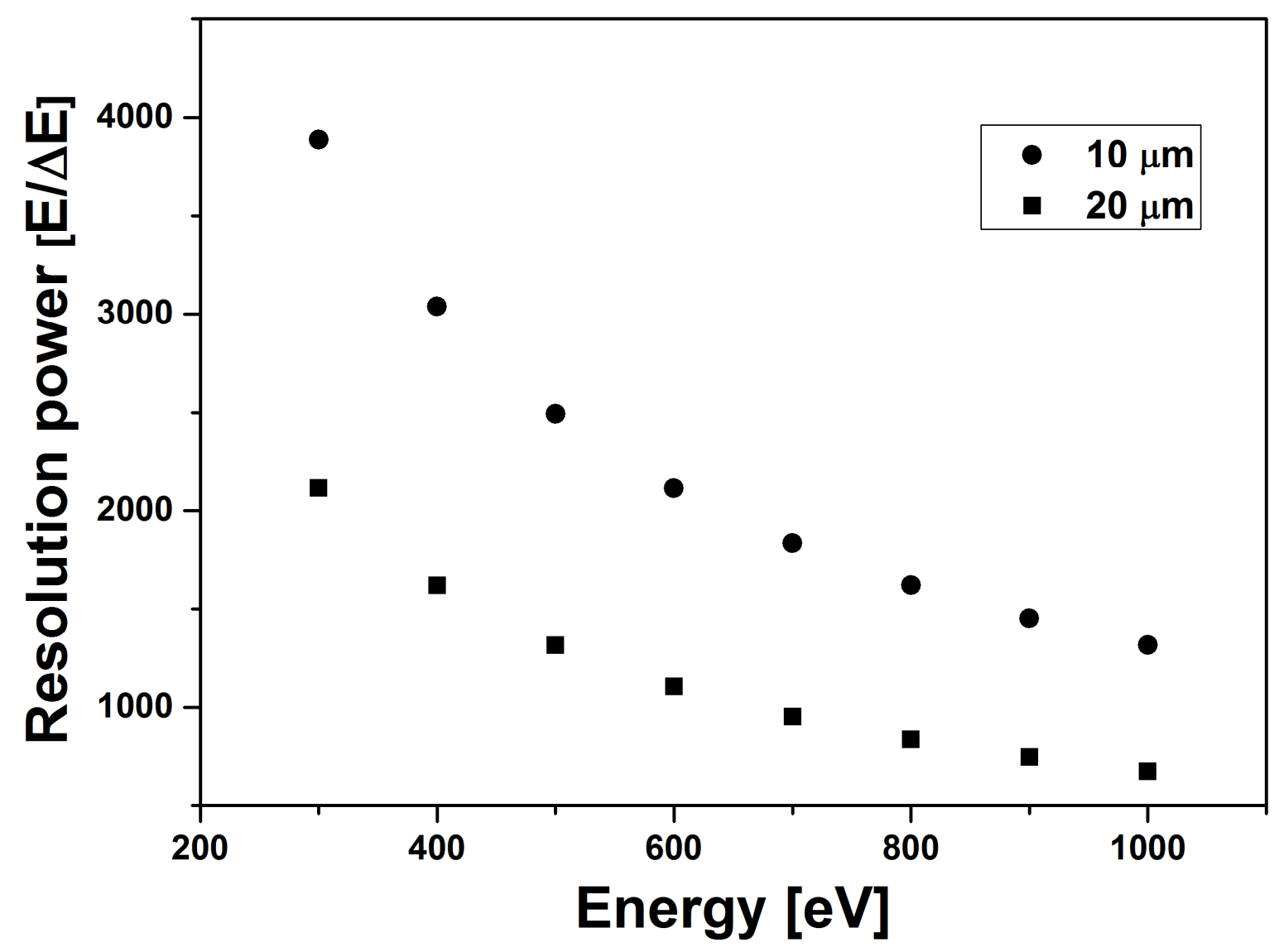

Fig. 4: The potential resolution power in dependence of the source/slit size.

\section{SUMMARY}

We designed a new endstation for XAS and high resolution XES and RIXS experiments at the most brilliant synchrotron facility to date, PETRA III. The experimental setup is easily extendable depending on the experimental conditions. The jet system can provide various samples in liquid phase to probe chemical and biological processes in their natural environment. A newly designed flat-field grating spectrometer records the fluorescence spectra of the systems. The endstation will be commissioned at the end of this year and will be available for users after the PETRA III extension.

\section{ACKNOWLEDGEMENTS}

This work was supported by SFB755 "Nanoscale Photonic Imaging” and SFB 1073 "Atomic Scale Control of Energy Conversion" of the German Science Foundation (DFG), the Advanced Study Group of the Max Planck Society, the Max Planck Society and DESY. Furthermore, S.T. is grateful to the Fonds of the Chemical Industry. We specially thank T. Dzelzainis, R. Reininger, F. Siewert, J. Rehanek, A. Hager, U. Hahn and J. Nordgren for their support. 


\section{REFERENCES}

[1] F. de Groot, "High-resolution X-ray emission and X-ray absorption spectroscopy," Chemical Reviews 101, 1779-1808 (2001).

[2] A. Föhlisch, P. Feulner, F. Hennies, A. Fink, D. Menzel, D. Sanchez-Portal, P. M. Echenique, and W. Wurth, "Direct observation of electron dynamics in the attosecond domain," Nature 436, 373-376 (2005) .

[3] M. Faubel, S. Schlemmer and J. P. Tönnies, "A molecular beam study of the evaporation of water from a liquid jet," Zeitschrift für Physik. D, Aoms, Molecules and Clusters 10, 269-277 (1988).

[4] O. Fuchs, F. Maier, L. Weinhardt, M. Weigand, M. Blum, M. Zharnikov, J. Denlinger, M. Grunze, C. Heske and E. Umbach, "A liquid flow cell to study the electronic structure of liquids with soft X-rays," Nuclear Instruments and Methods in Physics Research Section A: Accelerators, Spectrometers, Detectors and Associated Equipment 585, 172-177 (2008).

[5] B. Winter and M. Faubel, "Photoemission from Liquid Aqueous Solutions," Chemical Reviews 106, 1176-1211 (2006).

[6] I. Rajkovic, J. Hallmann, S. Grübel, R. More, W. Quevedo, M. Petri, and S. Techert, "Development of a multipurpose vacuum chamber for serial optical and diffraction experiments with free electron laser radiation.," The Review of scientific instruments 81, 045105 (2010).

[7] K. Kunnus, I. Rajkovic, S. Schreck, W. Quevedo, S. Eckert, M. Beye, E. Suljoti, C. Weniger, C. Kalus, S. Grübel, M. Scholz, D. Nordlund, W. Zhang, R. W. Hartsock, K. J. Gaffney, W. F. Schlotter, J. J. Turner, B. Kennedy, F. Hennies, S. Techert, P. Wernet, and A. Föhlisch, "A setup for resonant inelastic soft X-ray scattering on liquids at free electron laser light sources.," The Review of scientific instruments 83, 123109 (2012).

[8] K. M. Lange, R. Könnecke, S. Ghadimi, R. Golnak, M. a. Soldatov, K. F. Hodeck, A. Soldatov, and E. F. Aziz, "High resolution X-ray emission spectroscopy of water and aqueous ions using the micro-jet technique," Chemical Physics 377, 1-5 (2010).

[9] http://www.andor.com/scientific-cameras/high-energy-detection 